\title{
THE CENTER OF TOPOLOGICALLY PRIMITIVE EXPONENTIALLY GALBED ALGEBRAS
}

\author{
MART ABEL AND MATI ABEL
}

Received 29 September 2004; Revised 11 December 2005; Accepted 18 December 2005

Let $A$ be a unital sequentially complete topologically primitive exponentially galbed Hausdorff algebra over $\mathbb{C}$, in which all elements are bounded. It is shown that the center of $A$ is topologically isomorphic to $\mathbb{C}$.

Copyright (c) 2006 Hindawi Publishing Corporation. All rights reserved.

\section{Introduction}

(1) Let $A$ be an associative topological algebra over the field of complex numbers $\mathbb{C}$ with separately continuous multiplication. Then $A$ is an exponentially galbed algebra (see, e.g., $[1-4,19,20])$ if every neighbourhood $O$ of zero in $A$ defines another neighbourhood $U$ of zero such that

$$
\left\{\sum_{k=0}^{n} \frac{a_{k}}{2^{k}}: a_{0}, \ldots, a_{n} \in U\right\} \subset O
$$

for each $n \in \mathbb{N}$. Herewith, $A$ is locally pseudoconvex, if it has a base $\left\{U_{\lambda}: \lambda \in \Lambda\right\}$ of neighbourhoods of zero consisting of balanced and pseudoconvex sets (i.e., of sets $U$ for which $\mu U \subset U$, whenever $|\mu| \leqslant 1$, and $U+U \subset \rho U$ for a $\rho \geqslant 2$ ). In particular, when every $U_{\lambda}$ in $\left\{U_{\lambda}: \lambda \in \Lambda\right\}$ is idempotent (i.e., $U_{\lambda} U_{\lambda} \subset U_{\lambda}$ ), then $A$ is called a locally $m$-pseudoconvex algebra, and when every $U_{\lambda}$ in $\left\{U_{\lambda}: \lambda \in \Lambda\right\}$ is $A$-pseudoconvex (i.e., for any $a \in A$ there is a $\mu>0$ such that $a U_{\lambda}, U_{\lambda} a \subset \mu U_{\lambda}$ ), then $A$ is called a locally $A$-pseudoconvex algebra. It is well known (see [21, page 4] or [6, page 189]) that the locally pseudoconvex topology on $A$ is given by a family $\left\{p_{\lambda}: \lambda \in \Lambda\right\}$ of $k_{\lambda}$-homogeneous seminorms, where $k_{\lambda} \in(0,1]$ for each $\lambda \in \Lambda$. The topology of a locally $m$-pseudoconvex ( $A$-pseudoconvex) algebra $A$ is given by a family $\left\{p_{\lambda}: \lambda \in \Lambda\right\}$ of $k_{\lambda}$-homogeneous submultiplicative (i.e., $p_{\lambda}(a b) \leqslant p_{\lambda}(a) p_{\lambda}(b)$ for each $a, b \in A$ and $\lambda \in \Lambda$ ) (resp., $A$-multiplicative (i.e., for each $a \in A$ and each $\lambda \in$ $\Lambda$ there are numbers $N(a, \lambda)>0$ and $M(a, \lambda)>0$ such that $p_{\lambda}(a b) \leqslant N(a, \lambda) p_{\lambda}(b)$ and $p_{\lambda}(b a) \leqslant M(a, \lambda) p_{\lambda}(b)$ for each $\left.\left.b \in A\right)\right)$ seminorms, where $k_{\lambda} \in(0,1]$ for each $\lambda \in \Lambda$. In particular, when $k_{\lambda}=1$ for each $\lambda \in \Lambda$, then $A$ is a locally convex (resp., locally $m$-convex and locally A-convex) algebra and when the topology of $A$ has been defined by only one 
$k$-homogeneous seminorm with $k \in(0,1]$, then $A$ is a locally bounded algebra. It is easy to see that every locally pseudoconvex algebra is an exponentially galbed algebra.

Moreover, a complete locally bounded Hausdorff algebra $A$ is a $p$-Banach algebra; a complete metrizable algebra $A$ is a Fréchet algebra; a unital topological algebra $A$, in which the set of all invertible elements is open, is a Q-algebra (see, e.g., [14, page 43, Definition 6.2]) and a topological algebra $A$ is a topologically primitive algebra (see [5]), if

$$
\{a \in A: a A \subset M\}=\left\{\theta_{A}\right\} \quad\left(\{a \in A: A a \subset M\}=\left\{\theta_{A}\right\}\right)
$$

for a closed maximal regular (or modular) left (resp., right) ideal $M$ of $A$ (here $\theta_{A}$ denotes the zero element of $A$ ).

An element $a$ in a topological algebra $A$ is bounded, if there exists an element $\lambda_{a} \in$ $\mathbb{C} \backslash\{0\}$ such that the set

$$
\left\{\left(\frac{a}{\lambda_{a}}\right)^{n}: n \in \mathbb{N}\right\}
$$

is bounded in $A$ and nilpotent, if $a^{m}=\theta_{A}$ for some $m \in \mathbb{N}$. If all elements in $A$ are bounded (nilpotent), then $A$ is a topological algebra with bounded elements (resp., a nil algebra).

(2) It is well known that the center of a primitive ring (a ring (in particular, algebra) $R$ is primitive if it has a maximal left (right) regular ideal $M$ such that $\{a \in R: a R \subset M\}=$ $\left\{\theta_{R}\right\}$ (resp., $\left.\{a \in R: R a \subset M\}=\left\{\theta_{R}\right\}\right)$ ) is an integral domain (a ring $R$ is an integral domain, if from $a, b \in R$ and $a b=\theta_{R}$ follows that $a=\theta_{R}$ or $b=\theta_{R}$ ) (see [12, Lemma 2.1.3]) and any commutative integral domain can be the center of a primitive ring (see [13, Chapter II.6, Example 3]). Herewith, every field is a commutative integral domain, but any commutative integral domain is not necessarily a field. In particular (see [5]), when $R$ is a unital primitive locally $A$-pseudoconvex Hausdorff algebra over $\mathbb{C}$ or a unital locally pseudoconvex Fréchet $Q$-algebra over $\mathbb{C}$, then the center $Z(R)$ of $R$ is topologically isomorphic to $\mathbb{C}$ (for Banach algebras a similar result has been given in [16, Corollary 2.4.5] (see also [8, page 127], [15, Theorem 4.2.11], and [9, Theorem 2.6.26 (ii)]); for $k$-Banach algebras in [6, Corollary 9.3.7]; for unital primitive locally $m$-convex $Q$-algebras in [17, Corollary 2], and for unital primitive locally $A$-convex algebras, in which all maximal ideals are closed, in [18, Theorem 3]). For topological algebras with all maximal regular one-sided or two-sided ideals closed see also $[7,10,11,14]$.

In the present paper we will show that a similar result will be true for any unital sequentially complete topologically primitive exponentially galbed Hausdorff algebra over $\mathbb{C}$ in which all elements are bounded.

\section{Auxiliary results}

For describing the center of primitive exponentially galbed algebras we need the following results.

Proposition 2.1. Let $A$ be a unital exponentially galbed Hausdorff algebra over $\mathbb{C}$ with bounded elements, $\lambda_{0} \in \mathbb{C}$ and $a_{0} \in A$. If $A$ is a sequentially complete or a nil algebra, then 
there exists a neighbourhood $O\left(\lambda_{0}\right)$ of $\lambda_{0}$ such that

$$
\sum_{k=0}^{\infty}\left(\lambda-\lambda_{0}\right)^{k} a_{0}^{k}
$$

converges in $A$ and

$$
\left(e_{A}+\left(\lambda_{0}-\lambda\right) a_{0}\right)^{-1}=\sum_{k=0}^{\infty}\left(\lambda-\lambda_{0}\right)^{k} a_{0}^{k}
$$

for each $\lambda \in O\left(\lambda_{0}\right)$.

Proof. Let $O$ be an arbitrary neighbourhood of zero in $A$. Then there is a closed and balanced neighbourhood $O^{\prime}$ of zero in $A$ and a closed neighbourhood $O^{\prime \prime}$ of zero in $\mathbb{C}$ such that $O^{\prime \prime} O^{\prime} \subset O$. Now $O^{\prime}$ defines a balanced neighbourhood $V$ of zero in $A$ such that

$$
\left\{\sum_{k=0}^{n} \frac{v_{k}}{2^{k}}: v_{0}, \ldots, v_{n} \in V\right\} \subset O^{\prime}
$$

for each $n \in \mathbb{N}$. Since every element in $A$ is bounded, then there is a number $\mu_{0}=\mu_{a_{0}} \in$ $\mathbb{C} \backslash\{0\}$ such that

$$
\left\{\left(\frac{a_{0}}{\mu_{0}}\right)^{n}: n \in \mathbb{N}\right\}
$$

is bounded in $A$. Therefore, there exists a number $\rho_{0}>0$ such that

$$
\left(\frac{a_{0}}{\mu_{0}}\right)^{n} \in \rho_{0} V
$$

for each $n \in \mathbb{N}$.

Let now $a_{0} \in A$ and $\lambda_{0} \in \mathbb{C}$ be fixed,

$$
S_{n}(\lambda)=\sum_{k=0}^{n}\left(\lambda-\lambda_{0}\right)^{k} a_{0}^{k}
$$

for each $n \in \mathbb{N}$ and $\lambda \in \mathbb{C}$,

$$
U_{\mathbb{C}}=\left\{\lambda \in \mathbb{C}:|\lambda|<\frac{1}{3\left|\mu_{0}\right|}\right\}
$$

and $U\left(\lambda_{0}\right)=\lambda_{0}+U_{\mathbb{C}}$. Then

$$
S_{m}(\lambda)-S_{n}(\lambda)=\sum_{k=n+1}^{m}\left(\lambda-\lambda_{0}\right)^{k} a_{0}^{k}=\sum_{k=0}^{m-n-1}\left(\lambda-\lambda_{0}\right)^{n+k+1} a_{0}^{n+k+1}
$$

for each $n, m \in \mathbb{N}$, whenever $m>n$ and $\lambda \in \mathbb{C}$. If we take

$$
v_{n, k}(\lambda)=2^{k}\left(\lambda-\lambda_{0}\right)^{k} \frac{a_{0}^{n+k+1}}{\rho_{0} \mu_{0}^{n+1}}
$$


4 The center of exponentially galbed algebras

for each $n, k \in \mathbb{N}$ and $\lambda \in \mathbb{C}$, then

$$
S_{m}(\lambda)-S_{n}(\lambda)=\left(\lambda-\lambda_{0}\right)^{n+1} \mu_{0}{ }^{n+1} \rho_{0} \sum_{k=0}^{m-n-1} \frac{v_{n, k}(\lambda)}{2^{k}}
$$

for each $n, m \in \mathbb{N}$, whenever $m>n$ and $\lambda \in \mathbb{C}$. Now,

$$
v_{n, k}(\lambda)=\frac{1}{\rho_{0}}\left(2\left(\lambda-\lambda_{0}\right) \mu_{0}\right)^{k}\left(\frac{a_{0}}{\mu_{0}}\right)^{n+k+1} \in \frac{1}{\rho_{0}}\left(2 \mu_{0}\left(\lambda-\lambda_{0}\right)\right)^{k} \rho_{0} V \subset V
$$

for each $n, k \in \mathbb{N}$ and $\lambda \in U\left(\lambda_{0}\right)$, because $\left|2 \mu_{0}\left(\lambda-\lambda_{0}\right)\right|<2 / 3<1$. Hence,

$$
S_{m}(\lambda)-S_{n}(\lambda) \in \frac{\left(2 \mu_{0}\left(\lambda-\lambda_{0}\right)\right)^{n+1}}{2^{n+1}} \rho_{0} O^{\prime},
$$

whenever $m>n$ and $\lambda \in U\left(\lambda_{0}\right)$. Since again $\left|2 \mu_{0}\left(\lambda-\lambda_{0}\right)\right|<1$, then there exists a number $n_{0} \in \mathbb{N}$ such that

$$
\left(2 \mu_{0}\left(\lambda-\lambda_{0}\right)\right)^{n+1} \in \frac{1}{\rho_{0}} O^{\prime \prime}
$$

for each $n>n_{0}$. Taking this into account,

$$
S_{m}(\lambda)-S_{n}(\lambda) \in \frac{1}{2^{n+1}} \frac{1}{\rho_{0}} O^{\prime \prime} \rho_{0} O^{\prime} \subset O^{\prime \prime} O^{\prime} \subset O,
$$

whenever $m>n>n_{0}$ and $\lambda \in U\left(\lambda_{0}\right)$, since $O^{\prime}$ is balanced. It means that $\left(S_{n}(\lambda)\right)$ is a Cauchy complete, the sequence in $A$ for each $\lambda \in U\left(\lambda_{0}\right)$.

In the case when $A$ is sequentially complete the sequence $\left(S_{n}(\lambda)\right)$ converges in $A$. Suppose now that $A$ is a nil algebra. Then $a_{0}^{m+1}=\theta_{A}$ for some $m \in \mathbb{N}$. Hence,

$$
S_{n}(\lambda)=\sum_{k=0}^{m}\left(\lambda-\lambda_{0}\right)^{k} a_{0}^{k}
$$

for each $\lambda \in \mathbb{C}$, whenever $n \geq m$. Consequently, $\left(S_{n}(\lambda)\right)$ converges in $A$ for each $\lambda \in O\left(\lambda_{0}\right)$ in both cases.

Since

$$
\left(e_{A}+\left(\lambda_{0}-\lambda\right) a_{0}\right) \sum_{k=0}^{\infty}\left(\lambda-\lambda_{0}\right)^{k} a_{0}^{k}=\sum_{k=0}^{\infty}\left(\lambda-\lambda_{0}\right)^{k} a_{0}^{k}\left(e_{A}+\left(\lambda_{0}-\lambda\right) a_{0}\right)=e_{A},
$$

one gets

$$
\left(e_{A}+\left(\lambda_{0}-\lambda\right) a_{0}\right)^{-1}=\sum_{k=0}^{\infty}\left(\lambda-\lambda_{0}\right)^{k} a_{0}^{k}
$$

for each $\lambda \in O\left(\lambda_{0}\right)$. 
Corollary 2.2. Let $A$ be a unital exponentially galbed algebra over $\mathbb{C}$ with bounded elements. If $A$ is a sequentially complete or a nil algebra, then for each $a_{0} \in A$ there exists a number $R>0$ such that

$$
\sum_{k=0}^{\infty} \frac{a_{0}^{k}}{\mu^{k+1}}
$$

converges in $A$, whenever $|\mu|>R$.

Proof. If we take $\lambda_{0}=0$ in the previous proposition, then we get that

$$
\sum_{k=0}^{\infty} \lambda^{k} a_{0}^{k}
$$

converges in $A$, whenever $|\lambda|<\delta$ for some $\delta>0$. If now $\mu>R=\delta^{-1}$, then $\left|\mu^{-1}\right|<\delta$, which means that

$$
\sum_{k=0}^{\infty} \frac{a_{0}^{k}}{\mu^{k}}
$$

converges in $A$. Hence,

$$
\sum_{k=0}^{\infty} \frac{a_{0}^{k}}{\mu^{k+1}}=\frac{1}{\mu} \sum_{k=0}^{\infty} \frac{a_{0}^{k}}{\mu^{k}}
$$

converges in $A$, whenever $|\mu|>R$.

\section{Main result}

Now, based on Proposition 2.1 and Corollary 2.2, we give a description of the center $Z(A)$ of such unital topologically primitive exponentially galbed Hausdorff algebras $A$ over $\mathbb{C}$ in which all elements are bounded.

Theorem 3.1. Let $A$ be a unital sequentially complete topologically primitive exponentially galbed Hausdorff algebra over $\mathbb{C}$ with bounded elements. Then $Z(A)$ is topologically isomorphic to $\mathbb{C}$.

Proof. Since $A$ is a topologically primitive algebra, there is a closed maximal left ideal (if $M$ is a closed maximal right ideal, then the proof is similar) $M$ in $A$ such that

$$
\{a \in A: a A \subset M\}=\left\{\theta_{A}\right\}
$$

(then $M \cap Z(A)=\left\{\theta_{A}\right\}$ ). Denote by $\pi_{M}$ the canonical homomorphism from $A$ onto the quotient space $A-M$ of $A$ with respect to $M$. For each $z \in Z(A) \backslash\left\{\theta_{A}\right\}$ consider the left ideal

$$
K_{z}=\{a \in A: a z \in M\} .
$$

Since $m z=z m \in M$ for each $m \in M$ and $e_{A} z=z \notin M, M \subset K_{z} \neq A$. Hence, $K_{z}$ is a proper left ideal in $A$. Since the ideal $M$ is maximal, $M=K_{z}$ for each $z \in Z(A) \backslash\left\{\theta_{A}\right\}$. 
6 The center of exponentially galbed algebras

We will show that every $z \in Z(A)$ defines a number $\lambda_{z} \in \mathbb{C}$ such that $z=\lambda_{z} e_{A}$. If $z=\theta_{A}$, then we take $\lambda_{z}=0$. Suppose now that there exists a $z \in Z(A) \backslash\left\{\theta_{A}\right\}$ such that $z(\lambda)=$ $\lambda e_{A}-z \neq \theta_{A}$ for all $\lambda \in \mathbb{C}$. Then $z(\lambda) \in Z(A) \backslash\left\{\theta_{A}\right\}$ means that $z(\lambda) \notin M$ for each $\lambda \in \mathbb{C}$, $M+A z(\lambda)$ is a left ideal in $A, M \subset M+A z(\lambda)$ and

$$
z(\lambda)=\theta_{A}+e_{A} z(\lambda) \in(M+A z(\lambda)) \backslash M
$$

for each $\lambda \in \mathbb{C}$. Since $M$ is a maximal left ideal in $A$, then $M+A z(\lambda)=A$ for each $\lambda \in$ $\mathbb{C}$. Therefore, for each $\lambda \in \mathbb{C}$ there are elements $m(\lambda) \in M$ and $a(\lambda) \in A$ such that $e_{A}=$ $m(\lambda)+a(\lambda) z(\lambda)$, because of which $a(\lambda) z(\lambda)-e_{A} \in M$.

Let $a^{\prime}(\lambda) \in A$ be another element such that $a^{\prime}(\lambda) z(\lambda)-e_{A} \in M$. Then from

$$
\left[a(\lambda)-a^{\prime}(\lambda)\right] z(\lambda)=a(\lambda) z(\lambda)-a^{\prime}(\lambda) z(\lambda) \in M
$$

it follows that $\left[a(\lambda)-a^{\prime}(\lambda)\right] \in K_{z(\lambda)}=M$. Therefore, $\pi_{M}(a(\lambda))=\pi_{M}\left(a^{\prime}(\lambda)\right)$ for each $\lambda \in$ $\mathbb{C}$.

Let now $\lambda_{0} \in \mathbb{C}$ and

$$
d(\lambda)=e_{A}+\left(\lambda-\lambda_{0}\right) a\left(\lambda_{0}\right)
$$

for each $\lambda \in \mathbb{C}$. Then there is (by Proposition 2.1) a neighbourhood $O\left(\lambda_{0}\right)$ of $\lambda_{0}$ such that

$$
\sum_{k=0}^{\infty}\left(\lambda-\lambda_{0}\right)^{k} a\left(\lambda_{0}\right)^{k}
$$

converges in $A$ and

$$
d(\lambda)^{-1}=\sum_{k=0}^{\infty}\left(\lambda-\lambda_{0}\right)^{k} a\left(\lambda_{0}\right)^{k}
$$

for each $\lambda \in O\left(\lambda_{0}\right)$.

Now,

$$
\begin{aligned}
a\left(\lambda_{0}\right) & d(\lambda)^{-1} z(\lambda)-e_{A} \\
& =a\left(\lambda_{0}\right) d(\lambda)^{-1} z(\lambda)-\left[a\left(\lambda_{0}\right) z\left(\lambda_{0}\right)+m\left(\lambda_{0}\right)\right] \\
& =-a\left(\lambda_{0}\right) d(\lambda)^{-1}\left[-z(\lambda)+d(\lambda) z\left(\lambda_{0}\right)\right]-m\left(\lambda_{0}\right) \\
& =-a\left(\lambda_{0}\right) d(\lambda)^{-1}\left[\left(z-\lambda e_{A}\right)+\left(e_{A}+\left(\lambda-\lambda_{0}\right) a\left(\lambda_{0}\right)\right)\left(\lambda_{0} e_{A}-z\right)\right]-m\left(\lambda_{0}\right) \\
& =-a\left(\lambda_{0}\right) d(\lambda)^{-1}\left[\left(\lambda_{0}-\lambda\right)\left(e_{A}-a\left(\lambda_{0}\right) z\left(\lambda_{0}\right)\right)\right]-m\left(\lambda_{0}\right) \\
& =-a\left(\lambda_{0}\right) d(\lambda)^{-1}\left(\lambda_{0}-\lambda\right) m\left(\lambda_{0}\right)-m\left(\lambda_{0}\right) \in M .
\end{aligned}
$$

Therefore,

$$
\pi_{M}(a(\lambda))=\pi_{M}\left(a\left(\lambda_{0}\right) d(\lambda)^{-1}\right)
$$

for each $\lambda \in O\left(\lambda_{0}\right)$. 
Let now $\Psi(\lambda)=\pi_{M}(a(\lambda))$ for each $\lambda \in \mathbb{C}$. We will show that $\Psi$ is an $(A-M)$-valued analytic function (i.e., if $\lambda_{0} \in \mathbb{C}$, then there are a number $\delta>0$ and a sequence $\left(x_{n}\right)$ of elements of $A-M$ such that $\Psi\left(\lambda_{0}+\lambda\right)=\sum_{k=0}^{\infty}\left(x_{k} \lambda^{k}\right)$, whenever $|\lambda|<\delta$, and a number $R>0$ and a sequence $\left(y_{n}\right)$ of elements of $A-M$ such that $\Psi(\lambda)=\sum_{k=0}^{\infty}\left(y_{k} / \lambda^{k}\right)$, whenever $|\lambda|>R)$ on $\mathbb{C} \cup\{\infty\}$. For it, let again $\lambda_{0} \in \mathbb{C}$. Then $\Psi(\lambda)=\pi_{M}\left(a\left(\lambda_{0}\right) d(\lambda)^{-1}\right)$ for each $\lambda \in O\left(\lambda_{0}\right)$ and there exists a number $\delta>0$ such that $\lambda_{0}+\lambda \in O\left(\lambda_{0}\right)$, whenever $|\lambda|<\delta$. Now,

$$
\begin{aligned}
\Psi\left(\lambda_{0}+h\right) & =\pi_{M}\left(a\left(\lambda_{0}\right) d\left(\lambda_{0}+h\right)^{-1}\right) \\
& =\pi_{M}\left(a\left(\lambda_{0}\right) \sum_{k=0}^{\infty} h^{k} a\left(\lambda_{0}\right)^{k}\right)=\sum_{k=0}^{\infty} h^{k} \pi_{M}\left(a\left(\lambda_{0}\right)^{k+1}\right),
\end{aligned}
$$

if $|h|<\delta$, where $\pi_{M}\left(a\left(\lambda_{0}\right)^{k+1}\right) \in A-M$ for each $k \in \mathbb{N}$.

By Corollary 2.2, there is a number $R>0$ such that

$$
\sum_{k=0}^{\infty} \frac{z^{k}}{\lambda^{k+1}}
$$

converges in $A$, if $|\lambda|>R$. Easy calculation shows that

$$
z(\lambda) \sum_{k=0}^{\infty} \frac{z^{k}}{\lambda^{k+1}}=\sum_{k=0}^{\infty} \frac{z^{k}}{\lambda^{k+1}} z(\lambda)=e_{A} .
$$

Therefore,

$$
z(\lambda)^{-1}=\sum_{k=0}^{\infty} \frac{z^{k}}{\lambda^{k+1}}
$$

whenever $|\lambda|>R$. Since $z(\lambda)^{-1} z(\lambda)-e_{A} \in M$ for each $\lambda$ with $|\lambda|>R$, then

$$
\Psi(\lambda)=\pi_{M}\left(z(\lambda)^{-1}\right)=\pi_{M}\left(\sum_{k=0}^{\infty} \frac{z^{k}}{\lambda^{k+1}}\right)=\sum_{k=0}^{\infty} \frac{\pi_{M}\left(z^{k}\right)}{\lambda^{k+1}}
$$

if $|\lambda|>R$, where $\pi_{M}\left(z^{k}\right) \in A-M$ for each $k \in \mathbb{N}$. Consequently, $\Psi$ is an analytic $(A-M)$-valued function on $\mathbb{C} \cup\{\infty\}$. Since $A-M$ is an exponentially galbed Hausdorff space, $\Psi$ is a constant map by Turpin's theorem (see [19, page 56]).

We show that $\Psi(\lambda)=\theta_{A-M}$ for each $\lambda \in \mathbb{C}$. So, if $O$ is any neighbourhood of zero in $A$, then there exist in $A$ a closed neighbourhood $O^{\prime}$ of zero and a neighbourhood $V$ of zero such that $O^{\prime} \subset O$ and

$$
\left\{\sum_{k=0}^{n} \frac{v_{k}}{2^{k}}: v_{1}, \ldots, v_{n} \in V\right\} \subset O^{\prime}
$$

for each $n \in \mathbb{N}$. Moreover, there are $\mu_{z} \in \mathbb{C} \backslash\{0\}$ and $\rho_{V}>0$ such that

$$
\left(\frac{z}{\mu_{z}}\right)^{k} \in \rho_{V} V
$$


8 The center of exponentially galbed algebras

for each $k \in \mathbb{N}$. If now $|\lambda|>\max \left\{3\left|\mu_{z}\right|, \rho_{V}\right\}$, then

$$
v_{k}(\lambda)=\frac{2^{k} z^{k}}{\lambda^{k+1}}=\frac{1}{\rho_{V}} \frac{\rho_{V}}{\lambda}\left(\frac{2 \mu_{z}}{\lambda}\right)^{k}\left(\frac{z}{\mu_{z}}\right)^{k} \in \frac{1}{\rho_{V}}\left[\frac{\rho_{V}}{\lambda}\left(\frac{2 \mu_{z}}{\lambda}\right)^{k}\right] \rho_{V} V \subset V
$$

for each $k \in \mathbb{N}$. Therefore,

$$
\sum_{k=0}^{n} \frac{z^{k}}{\lambda^{k+1}}=\sum_{k=0}^{n} \frac{v_{k}(\lambda)}{2^{k}} \in O^{\prime}
$$

for each $n \in \mathbb{N}$. Since $O^{\prime}$ is closed, then

$$
z(\lambda)^{-1}=\sum_{k=0}^{\infty} \frac{z^{k}}{\lambda^{k+1}}=\lim _{n \rightarrow \infty} \sum_{k=0}^{n} \frac{v_{k}(\lambda)}{2^{k}} \in O^{\prime} \subset O,
$$

whenever $|\lambda|>\max \left\{3\left|\mu_{z}\right|, \rho_{V}, R\right\}$. Hence,

$$
\begin{gathered}
\lim _{|\lambda| \rightarrow \infty} z(\lambda)^{-1}=\theta_{A} \\
\lim _{|\lambda| \rightarrow \infty} \Psi(\lambda)=\lim _{|\lambda| \rightarrow \infty} \pi_{M}\left(z(\lambda)^{-1}\right)=\pi_{M}\left(\lim _{|\lambda| \rightarrow \infty} z(\lambda)^{-1}\right)=\theta_{A-M} .
\end{gathered}
$$

Thus, $\Psi(\lambda)=\theta_{A-M}$ or $a(\lambda) \in M$ for each $\lambda \in \mathbb{C}$. Therefore,

$$
e_{A}=-\left(a(\lambda) z(\lambda)-e_{A}\right)+a(\lambda) z(\lambda) \in M,
$$

which is a contradiction. Consequently, every $z \in Z(A)$ defines a $\lambda_{z} \in \mathbb{C}$ such that $z=$ $\lambda_{z} e_{A}$. Hence, $Z(A)$ is isomorphic to $\mathbb{C}$.

Moreover, the isomorphism $\rho$, defined by $\rho(z)=\lambda_{z}$ for each $z \in Z(A)$, is continuous. Indeed, if $O$ is a neighbourhood of zero in $\mathbb{C}$, then there exists an $\epsilon>0$ such that

$$
O_{\epsilon}=\{\lambda \in \mathbb{C}:|\lambda|<\epsilon\} \subset O .
$$

Let $\lambda_{0} \in O_{\epsilon} \backslash\{0\}$. Since $A$ is a Hausdorff space, there exists a balanced neighbourhood $V$ of zero in $A$ such that $\lambda_{0} e_{A} \notin V$. But then we also have

$$
\lambda_{0} e_{A} \notin V^{\prime}=V \cap Z(A) .
$$

If $\left|\lambda_{z}\right| \geqslant\left|\lambda_{0}\right|$, then $\left|\lambda_{0} \lambda_{z}^{-1}\right| \leqslant 1$ and $\lambda_{0} e_{A}=\left(\lambda_{0} \lambda_{z}^{-1}\right) z \in V^{\prime}$ for each $z \in V^{\prime}$, which is not possible. Hence, $\lambda_{z} \in O$ for each $z \in V^{\prime}$. Thus, $\rho$ is continuous ( $\rho^{-1}$ is continuous because $Z(A)$ is a topological linear space in the subspace topology). Consequently, $Z(A)$ is topologically isomorphic to $\mathbb{C}$. 
Remark 3.2. Using Theorem 3.1, it is possible to describe all closed maximal regular onesided and two-sided ideals in sequentially complete exponentially galbed algebras with bounded elements.

\section{Acknowledgments}

We would like to express our gratitude to the referee for valuable remarks and suggestions. Research is in part supported by Estonian Science Foundation Grant 6205.

\section{References}

[1] M. Abel, On the Gel'fand-Mazur theorem for exponentially galbed algebras, Tartu Riikliku Ülikooli Toimetised (1990), no. 899, 65-70.

[2] _ Gel'fand-Mazur algebras, Topological Vector Spaces, Algebras and Related Areas (Hamilton, ON, 1994), Pitman Research Notes in Mathematics Series, vol. 316, Longman Scientific \& Technical, Harlow, 1994, pp. 116-129.

[3] Mart Abel, Description of closed maximal ideals in Gelfand-Mazur algebras, General Topological Algebras (Tartu, 1999), Mathematical Studies (Tartu), vol. 1, Estonian Mathematical Society, Tartu, 2001, pp. 7-13.

[4] - Sectional representations of Gelfand-Mazur algebras, Scientiae Mathematicae Japonicae 54 (2001), no. 3, 441-448.

[5] M. Abel, The center of primitive locally pseudoconvex algebras, Bulletin of the Belgian Mathematical Society. Simon Stevin 11 (2004), no. 2, 191-199.

[6] V. K. Balachandran, Topological Algebras, North-Holland Mathematics Studies, vol. 185, NorthHolland, Amsterdam, 2000.

[7] B. A. Barnes, Modular annihilator algebras, Canadian Journal of Mathematics 18 (1966), 566578.

[8] F. F. Bonsall and J. Duncan, Complete Normed Algebras, Springer, New York, 1973.

[9] H. G. Dales, Banach Algebras and Automatic Continuity, London Mathematical Society Monographs. New Series, vol. 24, The Clarendon Press, Oxford University Press, New York, 2000.

[10] M. Haralampidou, Annihilator topological algebras, Portugaliae Mathematica 51 (1994), no. 1, $147-162$.

[11] - On complementing topological algebras. Functional analysis, Journal of Mathematical Sciences (New York) 96 (1999), no. 6, 3722-3734.

[12] I. N. Herstein, Noncommutative Rings, The Carus Mathematical Monographs, No. 15, John Wiley \& Sons, New York, 1968.

[13] N. Jacobson, Structure of Rings, Colloquium Publications, vol. 37, American Mathematical Society, Rhode Island, 1956.

[14] A. Mallios, Topological Algebras. Selected Topics, North-Holland Mathematics Studies, vol. 124, North-Holland, Amsterdam, 1986.

[15] Th. W. Palmer, Banach Algebras and the General Theory of *-Algebras. Vol. I: Algebras and Banach Algebras, Encyclopedia of Mathematics and Its Applications, vol. 49, Cambridge University Press, Cambridge, 1994.

[16] C. E. Rickart, General Topology of Banach Algebras, D. van Nostrad, New Jersey, 1960.

[17] Y. Tsertos, On primitive topological algebras, Bulletin of the Greek Mathematical Society 28 (1987), 81-92.

[18] On primitive A-convex algebras, Commentationes Mathematicae. Prace Matematyczne 41 (2001), 203-219.

[19] Ph. Turpin, Espaces et opérateurs exponentiellement galbés, Séminaire Pierre Lelong (Analyse), Année 1973-1974, Lecture Notes in Mathematics, vol. 474, Springer, Berlin, 1975, pp. 48-62. 
10 The center of exponentially galbed algebras

[20] Convexités dans les espaces vectoriels topologiques généraux, Dissertationes Mathematicae (Rozprawy Matematyczne) 131 (1976), 221.

[21] L. Waelbroeck, Topological Vector Spaces and Algebras, Lecture Notes in Mathematics, vol. 230, Springer, Berlin, 1971.

Mart Abel: Institute of Pure Mathematics, University of Tartu, J. Liivi 2-615, 50409 Tartu, Estonia E-mail address: mart.abel@ut.ee

Mati Abel: Institute of Pure Mathematics, University of Tartu, J. Liivi 2-615, 50409 Tartu, Estonia

E-mail address: mati.abel@ut.ee 


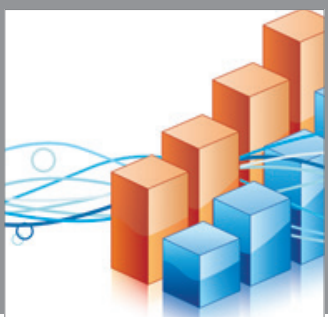

Advances in

Operations Research

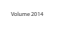

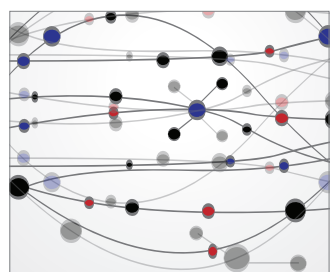

\section{The Scientific} World Journal
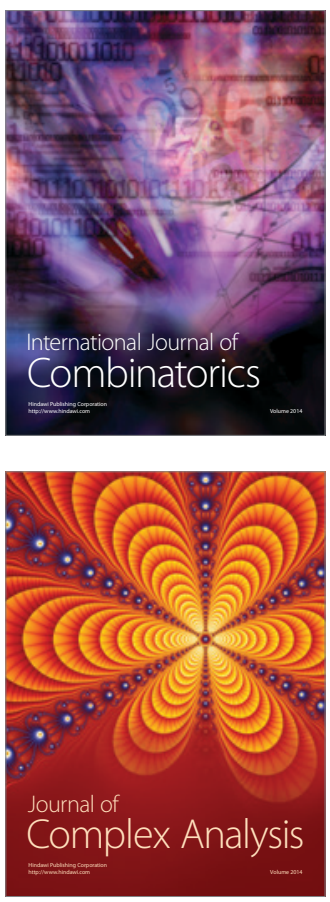

International Journal of

Mathematics and

Mathematical

Sciences
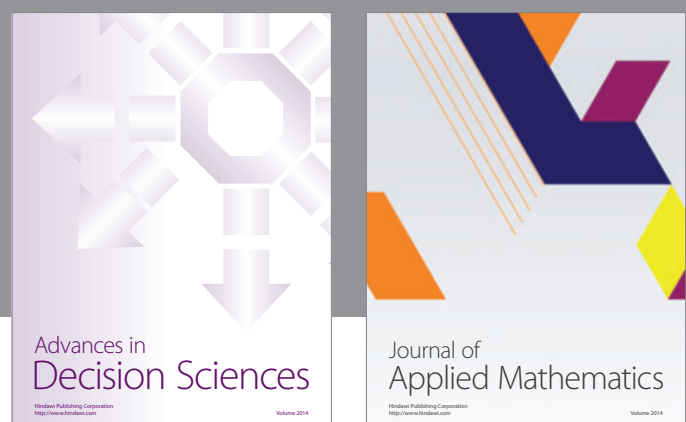

Journal of

Applied Mathematics
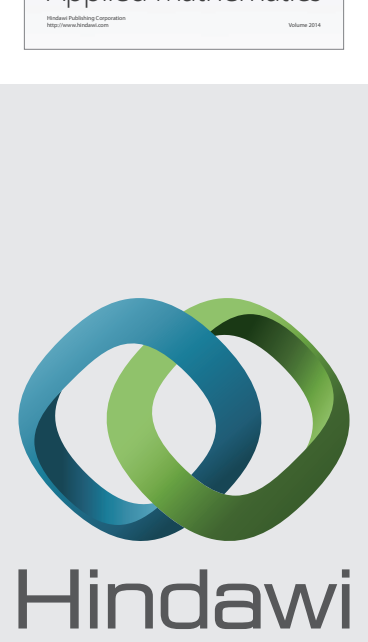

Submit your manuscripts at http://www.hindawi.com
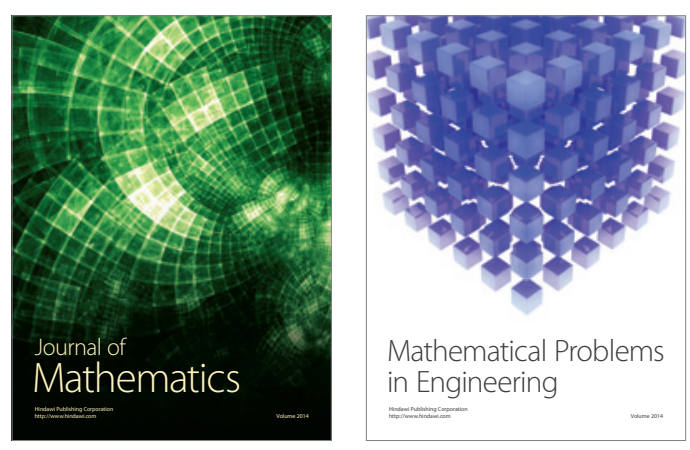

Mathematical Problems in Engineering
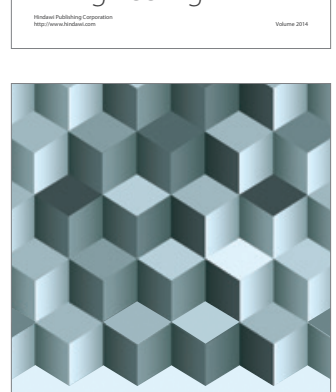

Journal of

Function Spaces
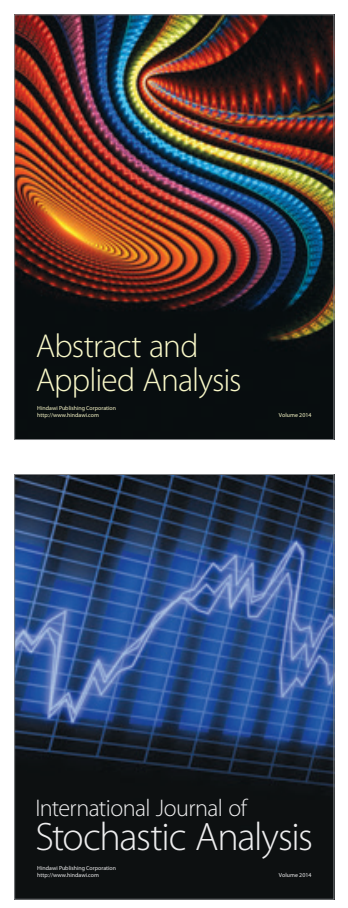

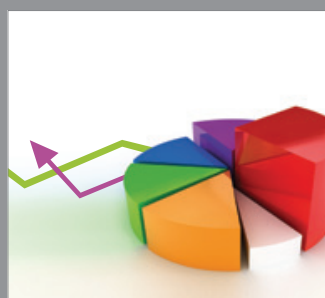

ournal of

Probability and Statistics

Promensencen
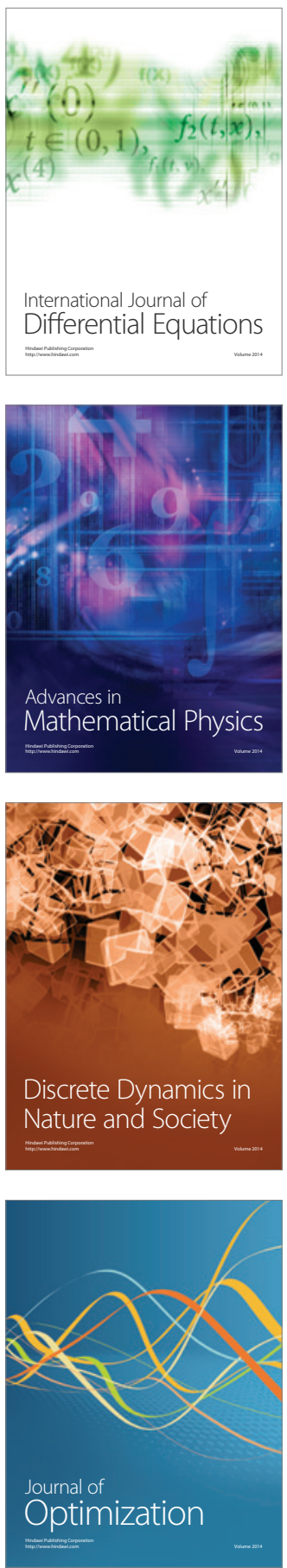